\title{
Congenital Tuberculosis
}

\author{
M. A. VOYCE and A. C. HUNT \\ From the Departments of Child Health and Pathology, University of Bristol
}

Tuberculosis in the first few weeks of life is now uncommon, but has a high mortality rate in spite of chemotherapy (Morison, 1963). This is a report of an infant who died at the age of 10 weeks, in whom the infection was probably acquired transplacentally.

\section{Case Report}

The infant was admitted at the age of 8 weeks with a history of pallor, vomiting, and failure to thrive. He was the first child born after an 8-year period of infertility. The mother, aged 28, was diagnosed as having a tuberculous pleural effusion in 1962, though no organisms were seen or cultured. An endometrial biopsy at this time, and another taken a year later, showed no evidence of tuberculosis. She was treated with streptomycin, PAS, and isoniazid for two years. In 1964, almost immediately after the end of therapy, she became unwell with pneumonia and pleurisy. This was thought not to be tuberculous and she recovered after tetracycline therapy. She was 5 months pregnant at the time. During the rest of pregnancy and in the puerperium she seemed in good health.

The baby was born at full term, birth weight $7 \mathrm{lb}$. 12 oz. $(3,515$ g.). The placenta appeared normal but was not examined histologically. He was well until he was 1 month when he began to vomit. At the age of 2 months he was pale and his abdomen was distended; his spleen was firm and extended to below the umbilicus and his liver was enlarged to three finger breadths below the costal margin. There were a few scattered small skin nodules each with an erythematous margin on the trunk and thighs. The axillary and inguinal lymph nodes were enlarged.

Investigation of the blood showed $\mathrm{Hb} 6 \cdot 4 \mathrm{~g} . / 100 \mathrm{ml}$., WBC $8,000 /$ c.mm. with 3,800 segmented cells $/$ c.mm. Leucocytes showed a left shift; reticulocytes $5.4 \%$; platelets $41,000 /$ c.mm. Bone-marrow examination showed active erythropoiesis and abundant megakaryocytes. The serum bilirubin was normal. Chest radiograph was normal.

A biopsy of one of the nodular skin lesions on the thigh showed a characteristic tuberculous granuloma in the upper dermis with an intact epidermis. An inguinal lymph node removed at the same time also showed tuberculous granulomas. Numerous acid-fast bacilli were demonstrated in the sections of the lesions.

Examination of the cerebrospinal fluid failed to show

Received October 18, 1965. any organisms and none were cultured. However, acidfast bacilli were later cultured from the stomach washing. These organisms were fully sensitive to streptomycin, PAS, and isoniazid.

Treatment was started with streptomycin, PAS, and isoniazid. In spite of this the child's condition deteriorated and the severe anaemia necessitated blood transfusion. At this stage Mantoux test $1: 1,000$ was negative. Fourteen days after admission he developed respiratory distress and died. A chest radiograph 48 hours before death showed slight mottling of all lung fields.

Although a limitation was placed on the extent of the necropsy, most of the thoracic and abdominal contents were examined.

The liver showed the most extensive and the largest lesions. These consisted of yellowish nodules between 0.2 and $0.4 \mathrm{~cm}$. diameter, and there were 2 or 3 such lesions in every square centimetre of cut surface. The spleen was almost as extensively involved but the individual nodules were slightly smaller. The lungs showed fewer, whiter, nodules less than $0.1 \mathrm{~cm}$. diameter, mostly on the pleural surfaces. The heart, stomach, intestines, and kidneys were normal to the naked eye.

Histology showed the typical appearances of tuberculosis with caseation. Many acid-fast bacilli were present in all lesions. In addition to those described above, occasional microscopical tubercles were found in the renal cortex and medulla.

After the death of the infant the mother was fully examined for evidence of active tuberculosis, without success. A further endometrial biopsy was carried out, and still no evidence of tuberculosis was present. There was no history of tuberculosis in anyone else who came in contact with the baby during his life.

\section{Discussion}

The diagnosis of tuberculosis in the mother was based on strong presumptive evidence, though the organism was not found. It is likely that following cessation of antituberculous therapy, there was a recrudescence of the disease at the fifth month of pregnancy, with transplacental spread. Corner and Brown (1955), Hughesdon (1946), and others have found that the mother of a baby with congenital tuberculosis usually has advanced tuberculosis herself, but this is not always so and sometimes no 
evidence of maternal tuberculosis has been found at the time of delivery. In some of these mothers tuberculous endometritis has been present (Riechle and Wheelock, 1939; Hertzog, Chapman, and Herring, 1949).

Tubercle bacilli may reach the foetus through the placenta and the umbilical vein, or through infected amniotic fluid. The severe involvement of the liver and spleen with less extensive lesions in the lung, in this instance, suggests the former method of spread. It must be emphasized, however, that the possibility of a postnatally acquired infection cannot be excluded, as the mother and child were not separated after birth. Extensive disease in the newborn from infection acquired after birth has been reported by Grady and Zuelzer (1955), but of their 5 patients 4 were in infants of very low birth weight and the other was exposed to a parent with 'far advanced T.B.' The severity of the infection, the distribution of the lesions, and the maternal history seem strong evidence to suggest an intrauterine source. The absence of tuberculosis in the endometrial biopsies before and after delivery suggests that the transplacental infection can occur without the presence of detectable lesions in the uterus.

Of clinical interest are the skin lesions and the enlarged lymph nodes. Todd (1960) first drew attention to the enlarged lymph nodes which he thought represented blood stream spread before birth. He also found absence of dissemination to the meninges. Of further importance is the fact that the acid-fast bacilli cultured from this infant were fully sensitive to the therapeutic agents used, in spite of the prolonged therapy that the mother had received. O'Donohoe (1963) has reported an infant who died at the age of 8 weeks of tuberculosis, the mother having had sarcoidosis in the past. She was treated with isoniazid, PAS, and prednisolone for a year, ending when she was one-month pregnant. The month after delivery the mother developed a tuberculous arthritis.

\section{Summary}

A 2-month-old infant with generalized tuberculosis is described. The mother had received a 2-year course of anti-tuberculous therapy which was discontinued during pregnancy.

We wish to thank the Departments of Child Health and Obstetrics for their assistance.

\section{REFERENCES}

Corner, B. D., and Brown, N. J. (1955). Congenital tuberculosis Report of a case with necropsy findings in mother and child. Thorax, 10, 99.

Grady, R. C., and Zuelzer, W. W. (1955). Neonatal tuberculosis. Amer. F. Dis. Child., 90, 381.

Hertzog, A. J., Chapman, S., and Herring, J. (1949). Congenital pulmonary aspiration-tuberculosis. Amer. F. clin. Path., 19, 1139.

Hughesdon, M. R. (1946). Congenital tuberculosis. Arch. Dis. Childh., 21, 121.

Morison, J. E. (1963). Foetal and Neonatal Pathology, 2nd ed. Butterworth, London.

O'Donohoe, N. V. (1963). Congenital tuberculosis and maternal sarcoidosis. Arch. Dis. Childh., 38, 83.

Riechle, H. S., and Wheelock, M. C. (1939). Aspiration type of congenital tuberculosis. Arch. Path., 28, 799.

Todd, R. McL. (1960). Congenital tuberculosis: report of a case with unusual features. Tubercle (Lond.), 41, 71. 